\title{
Effect of acute oral chlorogenic acid ingestion on the inhibition of blood glucose excursions following glucose tolerance testing
}

\author{
Shinichi Demura ${ }^{1}$, Takayoshi Yamada ${ }^{2}$, Yukio Hirose ${ }^{1}$, Kenji Takahashi $^{3}$ \\ ${ }^{1}$ Graduate School of Natural Science \& Technology, Kanazawa University, Kanazawa, Japan \\ ${ }^{2}$ General Education Center, Fukui National College of Technology, Fukui, Japan \\ ${ }^{3}$ Faculty of Community Health Care, Teikyo Heisei University, Ichihara, Japan \\ Email: demura@ed.kanazawa-u.ac.jp, takay@fukui-nct.ac.jp, hirose11@staff.kanazawa-u.ac.jp, kenji.takahashi@thu.ac.jp
}

Received 9 January 2013; revised 14 February 2013; accepted 28 February 2013

\begin{abstract}
The aim of this study was to examine the inhibitory effect of acute oral chlorogenic acid (CGA) ingestion on increases in blood glucose levels following glucose tolerance testing. Ten healthy male adults (age: $25.9 \pm$ 5.4 years) participated in the study. Blood samples were collected from the antecubital vein of subjects following overnight fasting. After a 120-min rest, they were administered $75 \mathrm{~g}$ glucose and chlorogenic acid or placebo. The amount of chlorogenic acid administered (in the form of capsules) to the subjects was 0.1 g per body mass. In addition, only capsules were ingested in placebo ingestion conditions. Blood samples were collected 4 times during the 120 -min rest period at intervals of $30 \mathrm{~min}$. Serum insulin and plasma glucose levels were analyzed. Serum insulin levels increased significantly at $\mathbf{3 0} \mathrm{min}$ after glucose ingestion, and fixed until $120 \mathrm{~min}$ in both conditions. Plasma glucose level increased significantly at $30 \mathrm{~min}$ after glucose ingestion, followed by a slow decrease. In addition, no significant difference was found between the conditions in each parameter. In conclusion, acute oral chlorogenic acid ingestion may not inhibition blood glucose increase following glucose tolerance.
\end{abstract}

Keywords: Chlorogenic Acid; Blood Glucose Level; Insulin Resistance; Glucose Tolerance Test

\section{INTRODUCTION}

Over 15 million people worldwide contract type 2 diabetes mellitus [1]. Diabetes is associated with a state of increased free radical production induced by chronic inflammation along with hyperglycemia, resulting in an imbalance between radical-generating and radical-scavenging systems [2]. Oxygen free radicals are known to be associated with a variety of cellular functions, but they can be both essential and highly toxic to cellular homeostasis [3]. Due to the damaging effects of increased oxidative stress observed during complications of diabetes mellitus, a countermeasure for relieving symptoms of diabetic complications and preventing the onset of symptoms is important [4].

Because type 2 diabetes is heterogeneous in nature, current therapies are unable to modify the natural history of this disorder. Therefore, it is necessary to explore new antidiabetic therapies. Polyphenol, an antioxidant found in many plants, fruits, vegetables, coffee, red wine, and tea, has been studied widely to examine its effect in reducing oxidative stress at the cellular level, thereby inhibiting the disease [5-7]. Coffee and wine are known to have a higher polyphenol content than other foods [8]. Until recently, it was reported that chlorogenic acid (CGA) inhibits blood glucose increase after a meal as well as reduces cholesterol and triacylglycerol levels; however, all prior studies examined the effect in vivo in rat models. Therefore, it is not clear if similar effects may be observed in human subjects $[9,10]$.

This study aimed to examine the effect of acute oral CGA ingestion on the inhibition of blood glucose increase following glucose tolerance.

\section{METHODS}

\subsection{Subjects}

Ten healthy young male adults without past type 2 diabetes history participated in this study (age: $25.9 \pm 5.4$ years; height: $171.2 \pm 6.1 \mathrm{~cm}$; body mass: $70.7 \pm 8.9 \mathrm{~kg}$ ). Written informed consent was obtained from all subjects after a full explanation of the experimental purpose and protocol was provided. Moreover, the experimental protocol was approved by the Ethics Committee on Human Experimentation of Faculty of Human Science, Kanazawa University (authorization number: 2012-10). 


\subsection{Experimental Design}

The experimental design was a double-blind, cross-over study. Subjects received both CGA and placebo ingestion over the course of the study, with a 1-week washout period. Moreover, the test condition order was counter balanced to eliminate order effect. In addition, subjects were instructed to refrain from intensive exercise for two days before the experiment and to fast the evening before the experiment. Subjects were also instructed to refrain from consuming beverages or food containing CGA and cold remedies.

\subsection{Experimental Condition}

CGA was ingested at a ratio of $0.1 \mathrm{~g} / \mathrm{kg}$ body weight. CGA is one of the polyphenol and their effect looks like tannin. It contains $5 \%-10 \%$ within coffee beans and its content is greater than caffeine ( $1 \%-2 \%)$. Antioxidative effect has been expected by its ability to entrap radical, and it is suggested to include the effect of delay glucose absorption. Both CGA and placebo were administered in capsules. Each capsules were administered with $75 \mathrm{~g}$ glucose (Partial hydrolysate of starch), which dissolved with $150 \mathrm{~g}$ water.

\subsection{Experimental Procedure and Protocol}

Subjects reported to the study laboratory twice during the experiment; the standard protocol for glucose tolerance testing was followed (Figure 1) with administration of glucose along with ingestion of either CGA or placebo at each visit. In addition, height, body weight, and body composition were measured at the first visit before engaging in the experimental protocol. Subjects rose at 7:00 hours following an overnight fast from 20:00 hours, and reported to the study lab. Approximately 30 minutes after reporting to the study lab, a baseline blood sample was obtained. The study subjects then were administered $75 \mathrm{~g}$ of glucose, which dissolved with $150 \mathrm{~g}$ water and CGA or a placebo capsules. Four blood samples were collected over a 120 -minute rest period at 30 min intervals.

\subsection{Parameters}

Blood samples collected over the 120 -min rest period

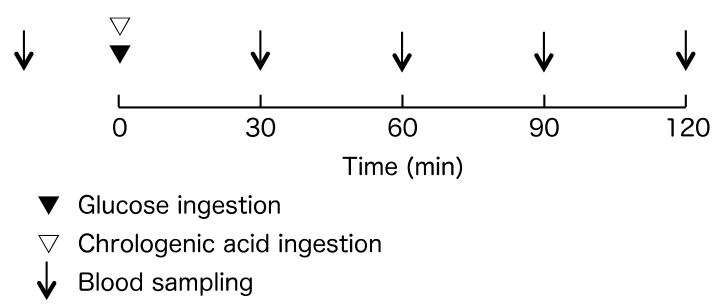

Figure 1. Experimental procedure. were analyzed for blood glucose and insulin. This was accomplished by transferring $3 \mathrm{~mL}$ of blood into a blood sampling tube containing sodium fluoride to assess blood glucose concentration and $7 \mathrm{~mL}$ of blood into a tube for analysis of insulin. These processes were carried out within $30 \mathrm{~s}$ of the blood collection. The samples were immediately centrifuged, and the supernatants were placed in chilled containers and stored at $-80^{\circ} \mathrm{C}$ until analyzed. Plasma glucose concentrations were analyzed by enzymatically. The inter-assay and intra-assay coefficients of variation $(\mathrm{CV})$ were $0.2 \%$ and $0.9 \%$, respectively. The samples for insulin were analyzed by high performance liquid chromatography (HPLC) using the HPLC system (Shimazu and Hitachi, Japan). Sensitivity, inter-assay, and intra-assay coefficients of variation $(\mathrm{CV})$ of this assay were $5.92 \mathrm{nmoL} / \mathrm{L}, 4.94 \%$, and $0.00 \%$, respectively.

\subsection{Statistical Analysis}

Two-way repeated measures analysis of variance (ingestion condition $\times$ measurement time) was used to examine the mean difference between CGA and placebo ingestion conditions for each parameter. When showing a significant main or interaction effect, Tukey's honestly significant difference (HSD) was used as post hoc analysis to examine specific mean differences. An alpha concentration of 0.05 was used for all experiments.

\section{RESULTS}

Figure 2 compares serum insulin concentration before $30,60,90$, and 120 min after administration of 75 g glucose along with either CGA or placebo. The significant main effect of time ( $\mathrm{F}=17.6, \mathrm{P}<0.001)$ was found, and serum insulin concentration on and after $30 \mathrm{~min}$ of glucose injection was significantly greater than that before ingestion in both conditions. No significant effect of ingestion and interaction were found $(\mathrm{F}=1.3, \mathrm{P}=0.291$

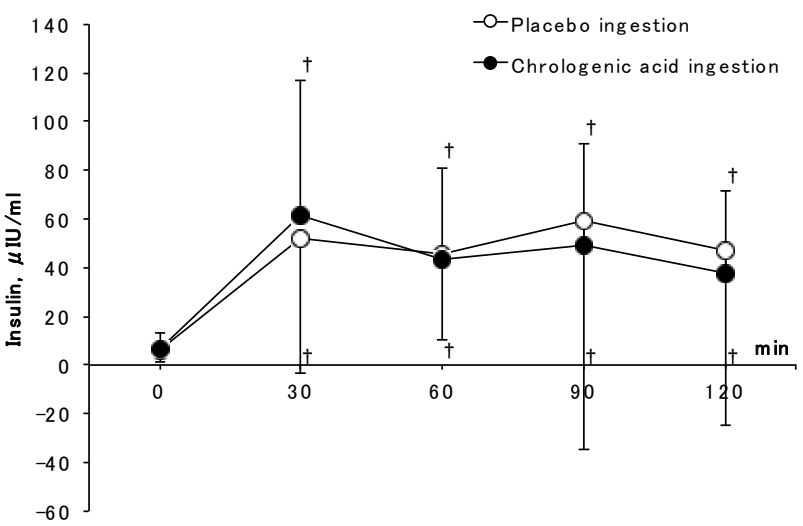

Figure 2. Serum insulin concentration before, 30, 60, 90 and 120 min after $75 \mathrm{~g}$ glucose ingestion in chlorogenic acid and placebo ingestion conditions. 'Shows significantly greater than baseline. 
and $\mathrm{F}=1.8, \mathrm{P}=0.177$ ). Figure 3 shows plasma glucose concentration before, $30,60,90$, and 120 min after glucose ingestion in the CGA and placebo ingestion conditions. A significant main effect of time $(\mathrm{F}=43.6, \mathrm{P}<$ 0.001 ) was observed, and plasma glucose concentration at 30 and $60 \mathrm{~min}$ after glucose ingestion was significantly greater than that before ingestion in both groups. No significant effect was observed on the ingestion conditions and interaction $(\mathrm{F}=2.4, \mathrm{P}=0.156$ and $\mathrm{F}=2.0, \mathrm{P}$ $=0.141$ ).

\section{DISCUSSION}

This study principally demonstrated that in human subjects, an ingestion of CGA does not necessarily lead to inhibition of blood glucose elevation or a decrease in blood insulin secretion following food ingestion in patients with type 2 diabetes mellitus. Until recently, it has been reported that CGA inhibits increases in blood glucose after a meal and decreases cholesterol and triacylglycerol levels $[9,10]$. However, these effects were observed in rats; therefore, it was essential to clarify whether similar effects are also observed in humans. For example, Nicasio et al. compared blood glucose concentration at 2 and 4 hours after glucose ingestion in subjects following CGA administration $(0.1,0.25,0.5,0.75$, and $1.0 \mathrm{~g} / \mathrm{kg}$ ) and placebo administration [9]. They reported that blood glucose concentration decreased significantly at 2 and 4 hours after glucose ingestion in subjects following CGA administration, and this decrease was directly proportional to the amount of CGA ingested. Meanwhile, Pari et al. examined the chronic effect of CGA ingestion $(5 \mathrm{mg} / \mathrm{kg})$ on blood glucose concentration of chemically-induced diabetic mice [10]. They reported that blood glucose concentration of chemically induced diabetic mice decreased significantly and insulin resistance was also improved by continuous CGA ingestion

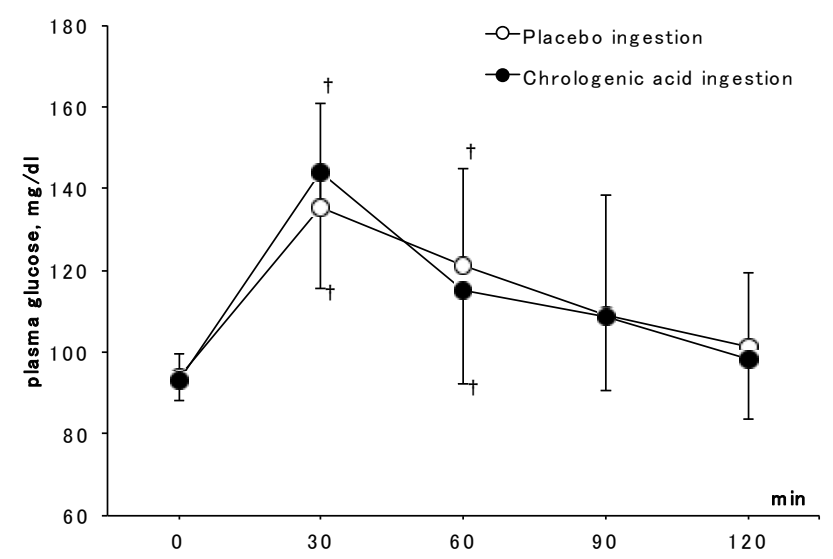

Figure 3. Plasma glucose concentration before, 30, 60, 90 and $120 \mathrm{~min}$ after $75 \mathrm{~g}$ glucose ingestion in chlorogenic acid and placebo ingestion conditions. ${ }^{\dagger}$ Shows significantly greater than baseline. for 45 days. Thus, there are many positive reports on the effect of CGA in inhibiting blood glucose in mice or rats. However, the present results, conducted on humans, differed from the aforementioned results. Nicasio et al. reported that blood glucose concentration decreased more with increased CGA ingestion [9]. However, a large decrease in blood glucose concentration was not found after a $0.1 \mathrm{~g} / \mathrm{kg}$ ingestion, which is similar to that in the present study. Moreover, their evaluation duration after glucose ingestion was longer than that in the present study (present study: 2 hours, Nicasio et al:: 4 hours). Thus, the effect of CGA ingestion in inhibiting blood glucose may not have been observed by the present volume of CGA ingested or the time of effect focused in this study. However, blood glucose increase was inhibited by longterm chlorogenic acid ingestion [10] and the ingestion volume $(5 \mathrm{mg} / \mathrm{kg})$ was considerably lower than that of the Nicasio et al. [9] and the present study. Therefore, a therapeutic effect requires ingestion of a larger amount of CGA. However, it is suggested that a similar inhibition of blood glucose can be gained by longterm ingestion with relatively little ingestion volume. Oxidative stress, the underlying mechanism behind diabetes and its complications, is induced as a result of a persistent and chronic state of imbalanced homeostatic mechanisms such as excessive body fluid volume, dysregulated salt sensitivity, and perturbations in the regulation of the renin-angiotensin system [4]. Meanwhile, free radicals, which are induced under the aforementioned conditions, are removed immediately under normal physiological conditions. However, a state of oxidative stress with excessive free radical formation breaks the state of balance between the creation and removal of free radicals. Therefore, considering the critical cause of diabetes, chronic free radical removal by long-term ingestion with a smaller volume may be a more useful coping strategy as compared with acute high amount of antioxidant ingestion. For a detailed effect of CGA, it will be necessary to examine the ingestion volume and the time period for the same. Although the present results did not show the decrease in insulin secretion with inhibition of blood glucose induced by CGA, it is suggested that the effects of the antioxidant in CGA, the ingestion volume, and the time period of the ingestion needs to be examined.

\section{REFERENCES}

[1] King, H., Aubert, R.E. and Herman, W.H. (1998) Global burden of diabetes, 1995-2025: Prevalence, numerical estimates, and projections. Diabetes Care, 21, 1414-1431. doi:10.2337/diacare.21.9.1414

[2] Baynes, J.W. (1991) Role of oxidative stress in the development of complication in diabetes. Diabetes, 40, 405 412. doi: $10.2337 /$ diabetes.40.4.405 
[3] Maritim, A.C., Sanders, R.A. and Watkins, J.B. (2003) Diabetes, oxidative stress, and antioxidants: A review. Journal of Biochemical and Molecular Toxicology, 17, 24-39. doi:10.1002/jbt.10058

[4] Izumi, Y., Matsumura, A., Wakita, S., Akagi, K., Fukuda, H., Kume, T., Irie, K., Takada-Takatori, Y., Sugimoto, H., Hashimoto, T. and Akaike, A. (2012) Isolation, identification, and biological evaluation of Nrf2-ARE activator from the leaves of green perilla (Perilla frutescens var. crispa f. viridis). Free Radical Biology and Medicine, 15, 669-679. doi:10.1016/i.freeradbiomed.2012.06.021

[5] Whaley-Connell, A., McCullough, P.A. and Sowers, J.R. (2011) The role of oxidative stress in the metabolic syndrome. Reviews in Cardiovascular Medicine, 12, 21-29.

[6] Basli, A., Soulet, S., Chaher, N., Mérillon, J.M., Chibane, M., Monti, J.P. and Richard, T. (2012) Wine polyphenols: Potential agents in neuroprotection. Oxidative Medicine and Cellular Longevity, 2012, Article ID: 805762. doi:10.1155/2012/805762
[7] Gramza-Michałowska, A. and Człapka-Matyasik, M. (2011) Evaluation of the antiradical potential of fruit and vegetable snacks. ACTA Scientiarum Polonorum Technologia Alimentaria, 10, 63-72.

[8] Burke, M.F., Khera, A.V. and Rader, D.J. (2010) Polyphenols and cholesterol efflux: Is coffee the next red wine? Circulation Research, 106, 627-629. doi:10.1161/CIRCRESAHA.109.215855

[9] Nicasio, P., Aguilar-Santamaria, L., Aranda, E., Ortiz, S. and Gonzalez, M. (2005) Hypoglycemic effect and chlorogenic acid content in two Cecropia species. Phytotherapy Research, 19, 661-664. doi:10.1002/ptr.1722

[10] Pari, L., Karthikesan, K. and Menon, V.P. (2010) Comparative and combined effect of chlorogenic acid and tetrahydrocurcumin on antioxidant disparities in chemical induced experimental diabetes. Molecular and Cellular Biochemistry, 341, 109-117. doi:10.1007/s11010-010-0442-5 